\title{
Small and Transient Effect of Cannabis Oil for Osteoarthritis-Related Joint Pain: A Case Report
}

\author{
Annie Wang, Anita Lo, Kiran Ubhi, and Thomas Cameron
}

Can J Hosp Pharm. 2021;74(2):156-8

\section{INTRODUCTION}

The Arthritis Society, a Canadian health charity, describes osteoarthritis (OA) as "a progressive disease of the whole joint that leads to breakdown of joint cartilage and underlying bone." $\mathrm{OA}$ can affect any joint in the body, with symptoms that differ from one individual to the next, the most common being joint pain, joint stiffness, and swelling. OA is the most common form of arthritis and currently affects more than $10 \%$ of the adult population in Canada. ${ }^{2}$ The prevalence of $\mathrm{OA}$ increases with age; as such, with aging of the Canadian population, the general prevalence of arthritis is expected to increase to $20 \%$ (or 7 million) by $2031 .{ }^{3}$ Consequently, there is a powerful impetus to find effective solutions to manage this condition.

Current pharmacological approaches to the treatment of chronic pain associated with OA rely primarily on longterm use of acetaminophen, nonsteroidal anti-inflammatory drugs (NSAIDs), opioids, and injectable corticosteroids, and may include other drugs such as gabapentin. Over-thecounter supplements, such as glucosamine and chondroitin, may be considered, given that they may provide symptomatic relief for some patients. ${ }^{4}$ The use of these medications has been the mainstay of therapy for quite some time; however, some patients continue to experience uncontrolled pain while taking the usual therapies. As such, cannabis and cannabinoids are now being investigated as adjuncts for the treatment of OA-related pain. ${ }^{5}$ The shift in therapeutic cannabis research is based on the realization that, among the diverse array of biological activities exhibited by cannabinoids, the endocannabinoid system has been shown to play a role in pain perception, as well as modulating inflammation. ${ }^{6,7}$ This report describes an elderly patient with severe OA of the hips, knees, and shoulders who used cannabis products, including cannabidiol (CBD) and tetrahydrocannabinol (THC), in an attempt to manage pain.

\section{CASE REPORT}

An 85-year-old woman with severe OA was admitted to a long-term care home on August 15, 2018.* Her OA was noted to be particularly severe in the left hip and knee, an assessment that was confirmed by radiography. She had difficulty with transfers secondary to her OA and was non-ambulatory; therefore, she used a wheelchair for mobilization. Her medical history included atrial fibrillation, type 2 diabetes mellitus, and open-angle glaucoma. She reported an allergy to penicillin. Medications before admission included apixaban $5 \mathrm{mg}$ twice daily, metoprolol $50 \mathrm{mg}$ twice daily, ramipril $5 \mathrm{mg}$ twice daily, atorvastatin $20 \mathrm{mg}$ daily, insulin glargine 22 units in the morning and 15 units at bedtime, and 1 drop of latanoprost $0.005 \%$ in each eye once daily at bedtime. Her pain control medications were acetaminophen $650 \mathrm{mg}$ daily, diclofenac gel 10\% (compounded) twice daily, gabapentin $300 \mathrm{mg} 3$ times daily, hydromorphone $2 \mathrm{mg}$ 4 times daily and $2 \mathrm{mg}$ hourly as needed (PRN), and fentanyl patch $75 \mu \mathrm{g} / \mathrm{h}$ every 3 days. The resident denied the use of any over-the-counter medications or natural health products.

Until December 2018, the resident was using hydromorphone $1 \mathrm{mg}$ PRN, 5 to 7 times monthly; at that time, however, her pain worsened, and PRN use of hydromorphone also increased substantially, up to 5 to 7 times daily. After evaluation at a cannabis clinic and subsequent assessment by the general practitioner, the resident was started on CBD Oil Drops (CannTrust Inc) at a concentration of $25 \mathrm{mg} /$ $\mathrm{mL}$. The initial dosage was $0.2 \mathrm{~mL}(5 \mathrm{mg})$ orally twice daily; the dose was titrated up by $0.2 \mathrm{~mL}$ every week to reach $3.0 \mathrm{~mL}$ (75 mg) orally twice daily in February 2019. Immediately upon the introduction of CBD oil, her use of PRN hydromorphone declined to 3 to 5 times daily. The patient noted that the CBD made her "sleepy sometimes", but no other adverse effects were reported. We were encouraged by the resident's reduced analgesic requirements, which were not accompanied by any self-reported change in perceived pain. However, the reduction in PRN use was transient, lasting only 1 week, and use of hydromorphone subsequently returned to the levels that were in use immediately before initiation of CBD oil (i.e., 5-7 times daily), even as the resident continued taking CBD oil at a dosage of $3.0 \mathrm{~mL}$ orally twice daily.

*The patient provided informed consent for publication of this report. 
After her admission to the long-term care facility, the resident was assessed several times according to the Pain Assessment Tool used in the facility, which was adapted from a tool used by the Fraser Health Authority. The scale ranges from 0 (no pain) to 5 (extreme pain). No pain rating was available for December 2018, when CBD oil was started. However, in August 2018, the occupational therapist reported that the resident had a lot of joint pain (as documented in the Recreation Record), and in September 2018, the resident rated her pain and discomfort level as 5 out of 5. Subsequent ratings in February and March 2019, after initiation of the CBD oil, were 4.25 and 3.5 out of 5 , respectively. Before starting CBD oil, the resident's participation in recreation activities was severely limited. However, in February and March 2019, she was able to join in most of the activities. In addition, there was improvement on the pain flowsheet, which is used to track pain intensity (on a scale of 0 to 5) before and after an intervention. For example, on 3 separate occasions in January 2019, the resident's pain was reduced from 3 to 1 an hour after administration of CBD oil. The reduced pain scores and increased activity level documented during these later assessments may have been attributable to a co-analgesic effect between the CBD oil and all of the resident's other pain medications.

Once the CBD oil was titrated to the desired dose in March 2019, THC oil $25 \mathrm{mg} / \mathrm{mL}$ was also trialled, at the urging of the family, at a dosage of $0.1 \mathrm{~mL} 3$ times daily. The THC oil was discontinued 1 week later because of selfreported adverse events, such as dizziness, unclear speech, and cognitive impairment. The resident stated "[she did] not want to feel that way again."

\section{DISCUSSION}

Pharmacological approaches to the treatment of OA pain have typically focused on NSAIDs and opioids. Treatment of OA pain usually follows a stepwise approach, beginning with topical and oral analgesics, then shifting to NSAIDs, injectable corticosteroids, and eventually opioids. ${ }^{4}$ Patients whose pain remains uncontrolled with conventional pain management therapies may turn to alternative interventions for pain management, such as cannabinoids.

OA can be quite debilitating, and patients must often use their prescribed medications for long periods, despite certain repercussions of long-term usage and potential drug interactions. For example, acetaminophen can cause drug-induced hepatotoxicity when administered at high doses or with concomitant alcohol consumption. ${ }^{8}$ Similarly, adverse cardiovascular, renal, gastrointestinal, and cerebrovascular risks have been observed with long-term NSAID use. ${ }^{9}$ Additionally, in elderly patients with multiple comorbidities and polypharmacy, there is a concern about increased risk of drug interactions between pain medications and other medications, such as warfarin and corticosteroids. ${ }^{10}$ Finally, opioids have a plethora of potential adverse effects, including significant risks of dependence, overdose, and drug interactions. ${ }^{11,12}$ The use of co-analgesics, such as CBD oil, may be opioid-sparing.

It is imperative that research be directed toward alternative interventions for chronic pain, such as cannabinoids and other cannabis products. In the case reported here, the patient's PRN use of hydromorphone declined substantially for a brief period after she started CBD oil, but subjective pain measures (i.e., changes in pain scale and activity level) showed a mild but more sustained improvement over time. Hence, clinicians should be open to the possibility of adding cannabinoids to current therapies for the management of chronic OA pain.

OA pain can manifest as a combination of nociceptive, neuropathic, and inflammatory pain, which explains why multitargeted pharmaceutical approaches are required in many cases. The body's endocannabinoid system has been shown to ameliorate all these modalities of pain subtypes. ${ }^{13}$ Studies with animal models have demonstrated that under the inflammatory physiological conditions seen in arthritic joints, certain receptors of the endocannabinoid system (i.e., cannabinoid receptors 1 and 2 [CB1 and CB2] and fatty acid amide hydrolase [FAAH] receptors) are expressed to a greater extent in the synovium of OA joints, including localized and upregulated expression of $\mathrm{CB} 1$ and $\mathrm{CB} 2$ receptors relative to control joints. ${ }^{14}$ Moreover, a significant component of OA pain and disease progression is related to increased local inflammatory states. Further investigations are required to elucidate these underlying mechanisms and perhaps provide more evidence for the clinical utility of cannabinoids; however, several barriers to such research remain. There have been few large-scale controlled clinical studies investigating the pain-alleviating effects of cannabinoids. Pharmacologically plausible reasons for the benefit of cannabinoids in the management of chronic pain, in combination with exploratory research on self-administration of cannabis by those with $\mathrm{OA}$ and chronic pain conditions, suggest avenues for more research. ${ }^{15}$ The Canadian Rheumatology Association ${ }^{16}$ has stated that medical cannabis is not an alternative to standard care but may provide symptomatic relief for some individuals (for example, in the case reported here, as an adjunct to fentanyl and hydromorphone).

Future investigations should take into consideration the low, yet highly variable oral bioavailability of CBD. Perhaps inhalation, mucosal administration, or administration in the form of lipid nanoparticles will lead to more accurate and reliable dosing. ${ }^{17}$ Excessively high doses of CBD can yield unpleasant adverse effects, such as major fatigue and dizziness; therefore, subsequent studies should be conducted to decipher what dose and titration method are appropriate, especially for elderly patients, and these studies should include careful monitoring for adverse events. Additionally, future studies should evaluate the clinical utility of CBD use for different populations, to determine who is most likely to benefit. 


\section{CONCLUSION}

This case report suggests the potential for adjunctive use of cannabinoids in managing OA pain. Positive outcomes from the use of CBD oil in this case included a small reduction in PRN hydromorphone use, a consistent post-dose reduction in pain, and slight improvement in terms of functionality and participation relative to the situation in the months before the patient started CBD treatment. The changes observed in this case might have resulted from a placebo effect; as such, it will be important for high-quality clinical trials to be conducted to evaluate the efficacy of cannabinoids in treating OA pain.

\section{References}

1. Osteoarthritis [webpage]. Arthritis Society; [updated 2017 Sep; cited 2019 Jun 25]. Available from: https://arthritis.ca/about-arthritis/ arthritis-types-(a-z)/types/osteoarthritis

2. Arthritis in Canada: an ongoing challenge. Health Canada; 2003 [cited $2021 \mathrm{Feb} 16$ ]. Available from: https://www.arthritisresearch.ca/ wp-content/uploads/Arthritis_in_Canada.pdf

3. O’Donnell S, Lagacé C, McRae L, Bancej C. Life with arthritis in Canada: a personal and public health challenge. Chronic Dis Inj Can. 2011; 31(3):135-6.

4. Singh JA, Noorbaloochi S, MacDonald R, Maxwell LJ. Chondroitin for osteoarthritis. Cochrane Database Syst Rev. 2015;(1):CD005614.

5. Perrot S, Trouvin AP. Cannabis for musculoskeletal pain and arthritis: evidence is needed. Joint Bone Spine. 2019;86(1):1-3.

6. Ulugol A. The endocannabinoid system as a potential therapeutic target for pain modulation. Balkan Med J. 2014;31(2):115-20.

7. Stockings E, Campbell G, Hall WD, Nielsen S, Zagic D, Rahman R, et al. Cannabis and cannabinoids for the treatment of people with chronic noncancer pain conditions: a systematic review and meta-analysis of controlled and observational studies. Pain. 2018;159(10):1932-54.

8. Lee WM. Drug-induced hepatotoxicity. N Engl J Med. 2003;349(5): 474-85.

9. Marcum ZA, Hanlon JT. Recognizing the risks of chronic nonsteroidal anti-inflammatory drug use in older adults. Ann Longterm Care. 2010;18(9):24-7.

10. Fulton MM, Riley Allen E. Polypharmacy in the elderly: a literature review. J Am Acad Nurse Pract. 2005;17(4):123-32.
11. Rivat C, Ballantyne J. The dark side of opioids in pain management: basic science explains clinical observation. Pain Rep. 2016;1(2):e570.

12. Vowles KE, McEntee ML, Julnes PS, Frohe T, Ney JP, Van Der Goes DN. Rates of opioid misuse, abuse, and addiction in chronic pain: a systematic review and data synthesis. Pain. 2015;156(4):569-76.

13. O'Brien M, McDougall JJ. Cannabis and joints: scientific evidence for the alleviation of osteoarthritis pain by cannabinoids. Curr Opin Pharmacol. 2018;40:104-9.

14. Barrie N, Manolios N. The endocannabinoid system in pain and inflammation: its relevance to rheumatic disease. Eur J Rheumatol. 2017;4(3): 210-8.

15. Ware MA, Adams H, Guy GW. The medicinal use of cannabis in the UK: results of a nationwide survey. Int J Clin Pract. 2005;59(3):291-5.

16. Canadian Rheumatology Association (CRA) position statement on medical cannabis use in rheumatic disease. Canadian Rheumatology Association; 2019 [cited 2019 Nov 20]. Available from: https://rheum. $\mathrm{ca} /$ resources/publications/canadian-rheumatology-association-cra -position-statement-on-medical-cannabis-use-in-rheumatic-disease/

17. Bruni N, Della Pepa C, Oliaro-Bosso S, Pessione E, Gastaldi D, Dosio F. Cannabinoid delivery systems for pain and inflammation treatment. Molecules. 2018;23(10):2478.

Annie Wang, BSc, is a candidate in the entry-to-practice Doctor of Pharmacy program (Class of 2021), Faculty of Pharmaceutical Sciences, The University of British Columbia, Vancouver, British Columbia.

Anita Lo, BScPharm, PharmD, is with the Pharmacy, Ridge Meadows Hospital, Maple Ridge, British Columbia.

Kiran Ubhi, BSP, PharmD, is with the Pharmacy, Ridge Meadows Hospital, Maple Ridge, British Columbia.

Thomas Cameron, BSc(Hons), PharmD, RPh, is with LMPS Pharmacy Residency Program, Vancouver, British Columbia.

Competing interests: None declared.

Address correspondence to:

Annie Wang

Faculty of Pharmaceutical Sciences

The University of British Columbia

2405 Wesbrook Mall

Vancouver BC V6T 1 Z3

email: anniewang@alumni.ubc.ca

Funding: None received. 